\title{
The flow of air in a moving and stationary permeable layer during drying of bulk building materials
}

\author{
Valery Borovkov ${ }^{1, *}$, Yulia Bryanskaya ${ }^{1}$, Aleksandra Ostyakova ${ }^{1,2}$, and Manana \\ Medzveliya $^{1}$ \\ ${ }^{1}$ Moscow state university of civil engineering, Yaroslavskoye shosse, 26, Moscow, Russia, 129337 \\ ${ }^{2}$ Water Problem Institute RAS, 3, Gubkina str., Moscow, 119333, Russia
}

\begin{abstract}
With the use of the equation of motion in a one-dimensional formulation, the flow in a stationary and moving permeable layer during its blowing is investigated. Resistance to the flow within the layer is assumed to be linear in Darcy. The thickness of the boundary layers was determined and the velocity distribution was obtained for various patterns of motion encountered in problems of drying and extraction.
\end{abstract}

In a number of technological processes of firing and drying of granulated granular materials, for example, in the production of building materials, gas or air is blown through a permeable layer of material moving in the apparatus on the belt of the conveyor, or sliding by gravity. To calculate the processes of mass transfer and heat exchange between the gas and the material, as well as with the walls of the apparatus, it is necessary to establish the kinematic characteristics of the gas flow blown through the permeable layer, depending on the characteristics of the flow that flows around the upper boundary of the layer (Fig. 1). A similar problem of the flow of a heavy permeable layer moving along an inclined wall under the influence of gravity can be encountered in the technology of washing of the finished product, extracting impurities, and the like.

When investigating flow characteristics in a permeable layer, we consider the motion of a homogeneoufs model gas (or liquid) occupying the entire volume of the permeable layer. A similar model is usually used in the solution of filtration problems [1]. In this paper, we consider cases when the motion of all particles of a heavy permeable layer occurs at the same rate, which does not depend on the velocity of the flowing gas or liquid flow. Therefore, we consider blowing (or flow) modes with moderate velocities that do not lead to erosion of the permeable layer and do not cause weighing and entrainment of its particles.

* Corresponding author: mgsu-hydraulic@yandex.ru 


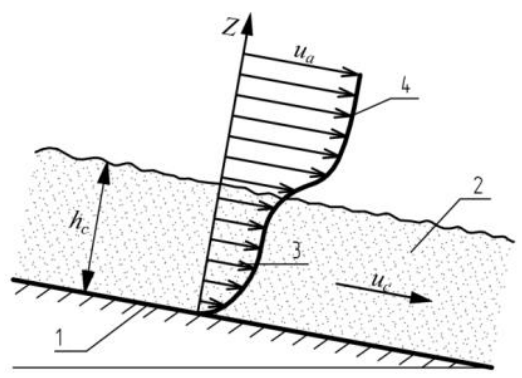

Fig. 1.

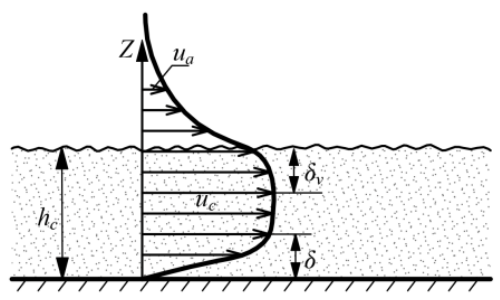

Fig. 2.

Fig. 1. The calculation scheme for blowing a permeable layer with a gas or a liquid. 1 - permeable bottom, 2 - layer of bulk material, 3 - velocity profile $u_{\mathrm{c}}, 4$ - gas or liquid velocity profile.

Fig. 2. The calculation scheme when airflow is absent.

The equation of uniform motion of the model environment (model fluid) within the permeable layer is written as:

$$
g i-\frac{d p}{\rho d x}+v_{\mathrm{e}} \frac{d^{2} u}{d z^{2}}+f_{\mathrm{g}}=0,
$$

where $g i$ - component of gravity; $\frac{d p}{d x}$ - longitudinal pressure gradient; $f_{\mathrm{g}}$ - specific force of filtration interaction between a model fluid and a solid permeable environment; $u$-longitudinal velocity of motion of a model environment in a fixed coordinate system associated with the wall; $v_{\mathrm{e}}-$ the effective viscosity of the model fluid.

In the formulation of this problem, great difficulties arise with the formulation of the hypothesis on the effective viscosity; for the Stokes flow regime, one of the problems was considered by Saffman [2]. The controversial nature of such hypotheses usually casts doubt on the acceptability of the approaches developed. Therefore, in this article an attempt is made to obtain solutions in parametric form with a parameter that depends on the effective viscosity, without a detailed deciphering of the factors determining it. The formulated problem, in the general case, is quite complicated, it allows us to consider a number of important special cases for practice.

Under conditions where the component of the weight force and the pressure gradient are absent, the movement of the model fluid is initiated by the intrinsic displacement of the permeable layer (Fig. 2). The equation of motion for this case takes the form:

$$
v_{\mathrm{e}}=\frac{d^{2} u}{d z^{2}}+f_{\mathrm{g}}=0
$$

Note that the force of interaction between a liquid and a permeable environment in this case is an active driving force. Despite the fact that the velocity of the motion of all the particles in the layer is the same according to the condition of the problem, the rate of the filtration motion of the liquid will change, since tangential stresses play an appreciable role at the wall, and interaction with the layer of the liquid at rest (or gas) at the outer boundary of the layer. The assumed linearity of the filtration flow excited in the permeable layer as it moves and, taking into account that the interfacial interaction force is proportional to the velocity of the gas or liquid relative to the permeable layer $\left(u_{\mathrm{c}}-u\right)$, force $f_{\mathrm{g}}$ we write as follows: 


$$
f_{\mathrm{g}}=g \frac{u_{\mathrm{c}}-u}{k_{\varphi}}
$$

where $k_{\varphi}$ - filtration coefficient.

Since the friction between the layers of the model fluid is related to the presence of the wall and is determined in the chosen coordinate system by the velocity gradient $u$ relative to the wall, the equation of motion takes the form:

$$
v_{\mathrm{e}} \frac{d^{2} u}{d z^{2}}+\frac{g\left(u_{\mathrm{c}}-u\right)}{k_{\varphi}}=0
$$

By introducing the effective permeability of a layer $k_{\mathrm{e}}=k_{\varphi} v_{\mathrm{e}} / g$ it reduces to the equation:

$$
\frac{d^{2} u}{d z^{2}}-\frac{u}{k_{\mathrm{e}}}=-F
$$

where $F=u_{\mathrm{c}} / k_{\mathrm{e}}$ - constant for the given case under consideration. This differential equation has a particular solution:

$$
u=k_{\mathrm{e}} F,
$$

and the general solution:

$$
u=C_{1} e^{z / \sqrt{k_{\mathrm{e}}}}+C_{2} e^{-z / \sqrt{k_{\mathrm{e}}}}
$$

Equation (5) thus has the form:

$$
u=C_{1} e^{z / \sqrt{k_{\mathrm{e}}}}+C_{2} e^{-z / \sqrt{k_{\mathrm{e}}}}+k_{\mathrm{e}} F
$$

To find the unknown coefficients $\mathrm{C}_{1}$ and $\mathrm{C}_{2}$, we can use the boundary conditions on the lower and upper boundary of the liquid layer $\delta$ (Fig. 2). From the boundary conditions in the form: at $z=0, u=0$; at $z=\delta, u=u_{\mathrm{c}}$, we get:

$$
C_{1}=\frac{u_{\mathrm{c}}-k_{\mathrm{e}} F+k_{\mathrm{e}} F e^{-\delta / \sqrt{k_{\mathrm{e}}}}}{e^{\delta / \sqrt{k_{\mathrm{e}}}}-e^{-\delta / \sqrt{k_{\mathrm{e}}}}}, C_{2}=\frac{u_{\mathrm{c}}-k_{\mathrm{e}} F+k_{\mathrm{e}} F e^{\delta / \sqrt{k_{\mathrm{e}}}}}{e^{\delta / \sqrt{k_{\mathrm{e}}}}-e^{-\delta / \sqrt{k_{\mathrm{e}}}}}
$$

Substituting the coefficients found in the solution of (8), taking into account that $e^{\delta / \sqrt{k_{\mathrm{e}}}}-e^{-\delta / \sqrt{k_{\mathrm{e}}}}=2 S h \delta / \sqrt{k_{\mathrm{e}}}$, since above the liquid layer under consideration $(z>\delta)$ derivatives $\frac{d u}{d z}=0$ и $\frac{d^{2} u}{d z^{2}}=0$ we find that $u_{\mathrm{c}}=k_{\mathrm{e}} F$. With this in mind, the solution looks like with the use of hyperbolic functions:

$$
\frac{u}{u_{\mathrm{c}}}=1-\frac{\operatorname{Sh}\left(z / \sqrt{k_{\mathrm{e}}}-\delta / \sqrt{k_{\mathrm{e}}}\right)}{\operatorname{Sh} \delta / \sqrt{k_{\mathrm{e}}}}
$$


The analysis shows that the profile obtained corresponds to a physically obvious condition $z=\delta, \frac{d u}{d z}=0$ only at $\delta \rightarrow \infty$, which agrees with the concepts of boundary-layer theory [3], [4], [5], [6], [7].

Approximate estimate of $\delta / \sqrt{k_{\mathrm{e}}}$, which is a parameter of the velocity profile, can be obtained by specifying a certain finite value of the velocity gradient at the outer boundary of the layer $\delta$. Considering the achievable accuracy of velocity measurements and taking the value of the velocity gradient at the upper boundary of the layer equal to $5 \%$ of the averaged gradient $u_{\mathrm{c}} / \delta$, we find an approximate estimate $\delta / \sqrt{k_{\mathrm{e}}} \approx 5$.

To determine the coefficients $C_{1}$ and $C_{2}$, being part of solution (8), we can also use the boundary conditions in the form: at $z=0, u=0$; at $z=\delta, \frac{d u}{d z}=0$. The resulting values of the coefficients have the form:

$$
C_{1}=-\frac{u_{\mathrm{c}} e^{-\delta / \sqrt{k_{\mathrm{e}}}}}{e^{\delta / \sqrt{k_{\mathrm{e}}}}+e^{-\delta / \sqrt{k_{\mathrm{e}}}}}, C_{2}=-\frac{u_{\mathrm{c}} e^{\delta / \sqrt{k_{\mathrm{e}}}}}{e^{\delta / \sqrt{k_{\mathrm{e}}}}+e^{-\delta / \sqrt{k_{\mathrm{e}}}}}
$$

Values $C_{1}$ and $C_{2}$ differ from the previously found expressions (9) and the velocity profile in the layer $\delta$ takes the form:

$$
\frac{u}{u_{\mathrm{c}}}=1-\frac{C h\left\lfloor\delta / \sqrt{k_{\mathrm{e}}}(1-z / \delta)\right\rfloor}{\operatorname{Ch} \delta / \sqrt{k_{\mathrm{e}}}}
$$

The resulting profile (12) provides a zero velocity gradient at $z=\delta$, but condition $u=u_{\mathrm{c}}$ is provided only with $\delta \rightarrow \infty$.

Approximate estimate $\delta$ in this case may be found from condition $u=0.97 u_{\mathrm{c}}$, as is usually done in boundary layer theory [3]. As is usually done in boundary layer theory (12) results in $\delta / \sqrt{k_{\mathrm{e}}} \approx 4$, which is close to the previous estimate $\delta / \sqrt{k_{\mathrm{e}}}$, obtained using the profile (10).

Comparison of the values of the velocity calculated from the profiles (10) and (12) shows that at $\delta / \sqrt{k_{\mathrm{e}}}=4$ they are sufficiently close to each other, which is connected with the smallness of the summand $e^{-\delta / \sqrt{k_{\mathrm{e}}}}$ in the expression for the hyperbolic sine and cosine. Given this, the velocity profile in the layer $\delta$ may be written in the form of the following simple expression:

$$
\frac{u}{u_{\mathrm{c}}}=1-e^{-z / \sqrt{k_{\mathrm{e}}}},
$$

fair in the above estimate $\delta / \sqrt{k_{\mathrm{e}}}$. Layer thickness $\delta$ in this case it may most simply be determined under viscous flow conditions in the wall layer. For other cases of flow, one or another hypothesis is required regarding the effective viscosity, the analysis and verification of which is the subject of a separate study. The approximate estimates obtained above for $\delta / \sqrt{k_{\mathrm{e}}}$ make it possible for us to establish that when the granular layer moves and the mean values of the filtration coefficient [8] for this case, the value $\delta$ turns out to be small and equal to several grain diameters. The value $\delta$ may be commensurable with the 
layer thickness $h_{\mathrm{c}}$ only in special cases with very high layer permeability and a small thickness $h_{\mathrm{c}}$.

At a layer thickness $h_{c} \gg \delta$, friction at its upper boundary will be manifested only in the layer $\delta_{v}$, adjacent to the upper boundary of the permeable layer being transported. The frictional force arising at the upper boundary will either slow the course of the layer (Fig. 3, a) or accelerate it (Fig. 3, b). The latter case is considered by V.N. Spiridonov [9].

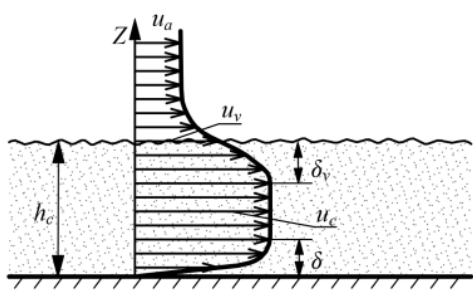

a)

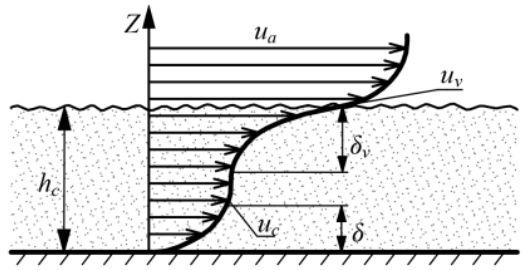

b)

Fig. 3. Calculation scheme, a) $u_{\mathrm{a}}>u_{\mathrm{c}}$, b) $u_{\mathrm{a}}<u_{c}$.

The above estimates for the layer thickness $\delta$ are apparently valid for the $\delta_{v}$ layer and allow us to assume that in most practically important cases for $u_{\mathrm{a}}>u_{\mathrm{c}}$ the velocity of the model fluid in the bulk layer will be equal to $u_{\mathrm{c}}$. Thus, the solutions obtained above for layer $\delta$ are retained for the given design case.

The flow in the layer $\delta_{v}$ for $u_{\mathrm{a}}<u_{c}$ will be similar to the considered flow in the layer $\delta$, therefore the solution (8) obtained in this case is also acceptable. The coefficients $C_{1}$ and $C_{2}$ can be determined from the conditions on the boundaries of the upper layer: $z=h_{\mathrm{c}}-\delta_{v}=z_{v}$, $u=u_{\mathrm{c}} ; z=h_{\mathrm{c}}, u=u_{v}$. Considering that $u_{\mathrm{c}}=k_{\mathrm{e}} F$, under these conditions, $C_{1}$ and $C_{2}$ may be determined for a known value of $u_{v}$. The velocity $u_{v}$ can be found from the consideration of the flow of a gas or liquid by the outer boundary of a layer. Thus, for example, if a turbulent boundary layer is formed in the external flow past a rough surface, a known logarithmic velocity profile can be used to determine $u_{v}$ [3], which for $u_{\mathrm{a}}<u_{\mathrm{c}}$ in the fixed coordinate system (see Figure 3) is written as follows:

$$
\frac{u_{\mathrm{c}}-u}{u_{*}}=\frac{1}{\kappa} \ln \frac{z^{\prime}}{k_{\mathrm{s}}}+8.48
$$

The coordinate $z^{\prime}$ is measured from the plane passing through the centers of the upper row of particles of the permeable layer; $k_{\mathrm{s}}$ is the equivalent sand roughness of the permeable layer; $u_{*}=\sqrt{\frac{\tau_{v}}{\rho}}$ is the dynamic velocity determined by the tangential stress $\tau_{v}$ at the upper boundary of the layer.

In the case when the blowing velocity $u_{\mathrm{a}}$ is greater than the velocity of the displacement of the layer $u_{\mathrm{c}}$ (Fig. 3, b), the velocity at the upper boundary of the layer is determined similarly from the profile (14). The dynamic velocity $u_{*}$ is determined from the calculation of the flow of gas or liquid in the upper part of the apparatus above the permeable layer. With known dimensions of this part of the apparatus and a given flow rate of gas or liquid, the value of $u_{*}$ is found from the well-known relationship: 


$$
\frac{u_{*}}{V}=\sqrt{\frac{\lambda}{8}},
$$

where $V$ is the average velocity of gas or liquid flow over the permeable layer; $\lambda_{v}-$ coefficient of hydraulic resistance, determined by the known methods of hydraulics [10].

It should be noted that in the case under consideration $\left(u_{\mathrm{a}}<u_{\mathrm{c}}\right)$ the positive increment of the shear stress $v_{\mathrm{e}} \frac{d^{2} u}{d z^{2}}$ is balanced by the interfacial interaction force $f_{\mathrm{g}}=g \frac{u-u_{\mathrm{c}}}{k_{\varphi}}$, and the equation of motion of the model fluid in the fixed coordinate system for this layer is written as:

$$
v_{\mathrm{e}} \frac{d^{2} u}{d z^{2}}-\frac{g\left(u-u_{\mathrm{c}}\right)}{k_{\varphi}}=0,
$$

which coincides with the already considered equation (4). Therefore: the solution (8) will also be suitable in this case also, taking into account the obvious peculiarities in determining the coefficients $C_{1}$ and $C_{2}$ on the basis of the corresponding boundary conditions. After finding the velocity profile in this layer, the estimate for the value $\delta_{v} / \sqrt{k_{\mathrm{e}}}$ that is a parameter of the velocity profile can be obtained, as before, on the basis of setting the velocity value or its gradient at the lower boundary of the layer in fractions of the average velocity gradient equal in this case $\frac{u_{v}-u_{\mathrm{c}}}{\delta_{v}}$.

In a number of practically important cases the motion of a model fluid can depend on the forces of gravity and the longitudinal pressure gradient $\frac{d p}{d x}$. The equation of motion is written as:

$$
g i-\frac{1}{\rho} \frac{d p}{d x}+v_{\mathrm{e}} \frac{d^{2} u}{d z^{2}}+f_{\mathrm{g}}=0
$$

where $g i$ - longitudinal component of gravity; $i$-slope of the wall of the apparatus.

Depending on the part of the permeable layer in which motion is considered, the interfacial interaction force $f_{\mathrm{g}}$, as well as the shear stress gradient, can change the sign, which should be taken into account in the calculations. Assuming, as before, the linear coupling of the force $f_{\mathrm{g}}$ with the flow velocity of particles of the granular layer, we write in the form:

$$
f_{\mathrm{g}}=\frac{g\left(u_{\mathrm{c}}-u\right)}{k_{\varphi}}
$$

For a wall layer $\left(u<u_{c}\right)$, the force $f_{g}>0$ is attractive and must enter the equation (17) with a positive sign.

$$
g i-\frac{1}{\rho} \frac{d p}{d x}+g \frac{u_{\mathrm{c}}}{k_{\varphi}}+v_{\mathrm{e}} \frac{d^{2} u}{d z^{2}}-g \frac{u}{k_{\varphi}}=0
$$


Determining $\frac{1}{v_{\mathrm{e}}}\left(g i-\frac{1}{\rho} \frac{d p}{d x}+g \frac{u_{\mathrm{c}}}{k_{\varphi}}\right)=F$, we write the equation of motion (17) in the form:

$$
\frac{d^{2} u}{d z^{2}}-\frac{1}{k_{\mathrm{e}}} u=-F
$$

The form of equation (18) coincides with the form of equation (5) considered earlier, and the solution of equation (5) is also acceptable in this case.

As before, the value $\delta / \sqrt{k_{\mathrm{e}}}$ may be found approximately for a given $\mathrm{F}$ from the condition of a definite value of the velocity gradient at the upper boundary of the layer. Using the boundary conditions $z=0, u=0 ; \mathrm{z}=\delta, \frac{d u}{d z}=0$ the velocity profile is written similarly to (12) as:

$$
\frac{u}{k_{\mathrm{e}} F}=1-\frac{C h \frac{\delta-z}{\sqrt{k_{\mathrm{e}}}}}{C h \delta / \sqrt{k_{\mathrm{e}}}}
$$

It should be noted that in the case where the liquid or gas moves in the apparatus under the action of a pressure gradient as well as gravity, the flow velocity $u_{\mathrm{c}}$ within the permeable layer will generally be less than the velocity $u_{\mathrm{a}}$ above the permeable layer due to the considerable resistance of the layer (see Figure 3). However, if the blowing speed $u_{\mathrm{a}}$ is small and the velocity of the layer $u_{\mathrm{c}}$ in the apparatus is considerable, the flow scheme shown in Fig. 3, a.

If the permeability of the layer is large at a small thickness, the flow cases shown in Fig. 4. When calculating the flow pattern (Fig. 4, a), the use of boundary conditions $z=0, u=0$; $z=\delta, u=u_{\mathrm{c}} ; z=h_{\mathrm{c}}-\delta_{v}=z_{v}, u=u_{\mathrm{c}}$ perhaps, however, the condition: at $\mathrm{z}=\delta, \frac{d u}{d z}=0$ should not be executed. Equality of derivatives $\left(\frac{d u}{d z}\right)_{z=\delta}$ For the velocity profiles in the layer $\delta$ and in the layer $\delta_{v}$ it ensures the smoothness of the function $u=f(z)$ at $z=\delta$. In the case when the velocity $u_{\mathrm{c}}$ is high at high layer permeability and its small thickness, it is possible that the maximum velocity will be less than $u_{c}$ (Fig. $4, \mathrm{~b}$ ). Then, as the boundary conditions, the following should be used:

$$
u=\text { idem } \text { at } z=\delta ;\left(\frac{d u}{d z}\right)_{z=\delta}=0
$$

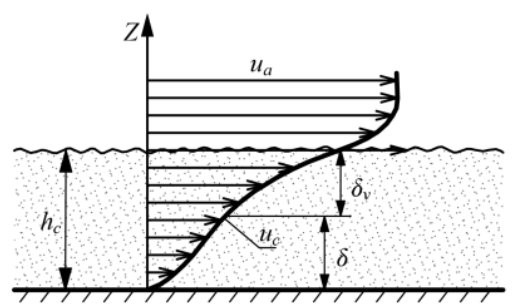

a)

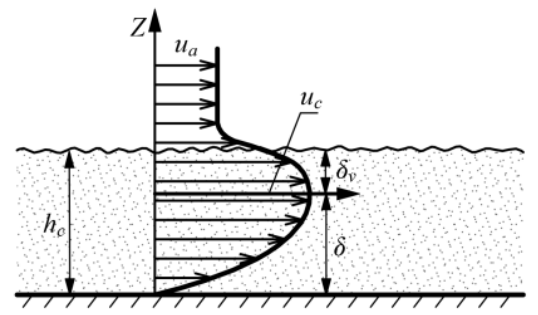

b)

Fig. 4. Calculation scheme for a small thickness of the permeable layer, a) $u_{\mathrm{a}}>u_{\mathrm{c}}$, b) $u_{\mathrm{a}}<u_{c}$. 
We note that the solutions and estimates obtained above are also valid for the special case when the permeable layer is fixed relative to the wall of the apparatus (at $u_{c}=0$ ). Thus, the analysis carried out made it possible to establish flow characteristics in the permeable layer of bulk material during its blowing and to obtain data that can be useful in solving drying and extraction problems in the production of building materials.

\section{References}

1. P. Ya. Polubarinova-Kochina, Theory of groundwater movement (Nauka, Moscow, 1977)

2. P.G. Saffman, Stadies in Applied Mathematics, Vol. 50, 2, p. 93 (1971)

3. G. Schlichting, The theory of the boundary layer (Science, Moscow, 1969)

4. Handbook of hydraulic calculations. Ed. P. G. Kiselev. (Energia, Moscow, 1972)

5. V. N. Spiridonov, Interaction between channel flow and filtration flow in disconnected soils (WPI RAS, Moscow, 1983)

6. A. D. Altshul, Hydraulic resistance (Nedra, Moscow, 1982)

7. V. V. Kafarov, Osnovy massoperedachi (Vysshaya shkola, Moscow, 1972)

8. G. Wallis, One-dimensional two-phase flows (Mir, Moscow, 1972)

9. Yu. V. Bryanskaya, I. M. Markova, A. V. Ostyakova, Hydraulics of the water flows and flows with a suspension in a rigid and a deformable boundaries, Ed. V. S. Borovkov (ASV, Moscow, 2009)

10. Bryanskaya Yu.V. Refinement of velocity profile parameters for flows in smooth and rough pipes, International Journal of Applied Engineering Research, Volume 11, 3, pp. 1694-1698 (2016) 